\title{
Processus territoriaux et gestion de l'eau en Camargue gardoise
}

\author{
Daniel Petit et Anne Rivière-Honegger
}

\section{Q OpenEdition}

Journals

Édition électronique

URL : http://journals.openedition.org/developpementdurable/1781

DOI : 10.4000/developpementdurable.1781

ISSN : 1772-9971

Éditeur

Association DD\&T

Référence électronique

Daniel Petit et Anne Rivière-Honegger, «Processus territoriaux et gestion de l'eau en Camargue gardoise », Développement durable et territoires [En ligne], Dossier 6 | 2006, mis en ligne le 08 janvier 2013, consulté le 20 avril 2019. URL : http://journals.openedition.org/developpementdurable/1781 DOI : 10.4000/developpementdurable.1781

Ce document a été généré automatiquement le 20 avril 2019.

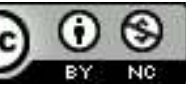

Développement Durable et Territoires est mis à disposition selon les termes de la licence Creative Commons Attribution - Pas d'Utilisation Commerciale 4.0 International. 


\title{
Processus territoriaux et gestion de l'eau en Camargue gardoise
}

\author{
Daniel Petit et Anne Rivière-Honegger
}

1 Le suivi de l'évolution du système de l'eau suppose une attitude de veille fort bien exprimée par R. Arrus: «Des éléments bougent, des mutations se préparent, des évolutions s'amplifient ou au contraire s'atténuent. Être attentifs à ces frémissements, les suivre dans leur durée, les estimer à défaut de les mesurer, c'est questionner à la fois les conflits engendrés au sein des rapports homme/nature et un futur possible qui sans cesse se décompose et se recompose » (Arrus, 2002, p. 9). C'est ce que nous faisons depuis plus de quinze ans dans le cadre d'une recherche appliquée en Camargue gardoise. Partie occidentale du delta du Rhône, ce vaste complexe de zones humides s'étend sur 38000 hectares et comprend huit communes qui comptent près de 45000 habitants.

2 La démarche méthodologique allie l'inventaire des documents existants auprès des acteurs exploitant le milieu, des différentes administrations et services concernés, à une reconnaissance détaillée du terrain et à des enquêtes directes auprès des interlocuteurs représentatifs des groupes identifiés. La cartographie est un moyen privilégié de restitution des résultats et des évolutions constatées.

Dans la réflexion sur les territoires de l'eau, cet espace est particulièrement intéressant par sa grande réactivité. Il s'agit, après avoir montré que la Camargue gardoise recèle une diversité de milieux et d'activités humaines en fortes interactions, de retracer l'histoire récente de la question du partage et de la gestion de l'eau qui ne cesse de défier les sociétés locales. Dans la mouvance des grandes mutations des années 1960-1970 en Camargue gardoise et face à l'obsolescence du Traité des Marais qui réglait la gestion collective des niveaux d'eau, les acteurs économiques liés à l'eau se sont positionnés sur des logiques individualistes. L'eau est devenue l'objet de maints conflits avec de graves conséquences sur les ressources et les milieux. La mise en œuvre d'une Charte de l'environnement et d'un SAGE au début des années 1990 a constitué les prémices du retour du «collectif » dans la gestion de l'eau. Mais on est encore loin de l'eau comme vecteur d'une construction territoriale à travers une communauté de projets liée à l'eau. 
Dans ce contexte, les crues de 2002 et 2003 sont apparues comme des révélateurs des inadéquations territoriales en cas de gestion de crise. Elles montrent que les politiques de l'eau ne peuvent faire l'impasse d'une réflexion sur l'articulation entre les différents espaces de décision. Sur quelles bases conduire cette réflexion? Les débats actuels vontils déboucher sur la création de nouveaux territoires de l'eau?

\section{Une diversité de milieux et d'activités humaines en fortes interactions}

4 La Camargue gardoise, ou Petite Camargue, est une zone humide à forte valeur patrimoniale attestée par de nombreuses reconnaissances, labels et inventaires: site inscrit (1963), ZICO ${ }^{1}$, inscription à l'inventaire Ramsar (1996) ${ }^{2}, \mathrm{ZNIEFF}^{3}$ (26 \% de la surface totale sont couverts par 2 ZNIEFF de type I), site test du programme MedWet ${ }^{4}$, site Natura $2000^{5}$ etc. En plus de leur valeur paysagère unique, de vastes roselières accueillent des oiseaux d'eau remarquables comme le Héron pourpré, le Butor étoilé, le Blongios nain ou le Crabier chevelu, la plupart menacés ${ }^{6}$. Plateforme de migration pour les oiseaux, le delta du Rhône ne dispose au sud de l'Europe que de deux zones équivalentes: le delta du Danube et celui du Guadalquivir.

Figure 1 : carte de situation et des territoires de gestion de l'eau

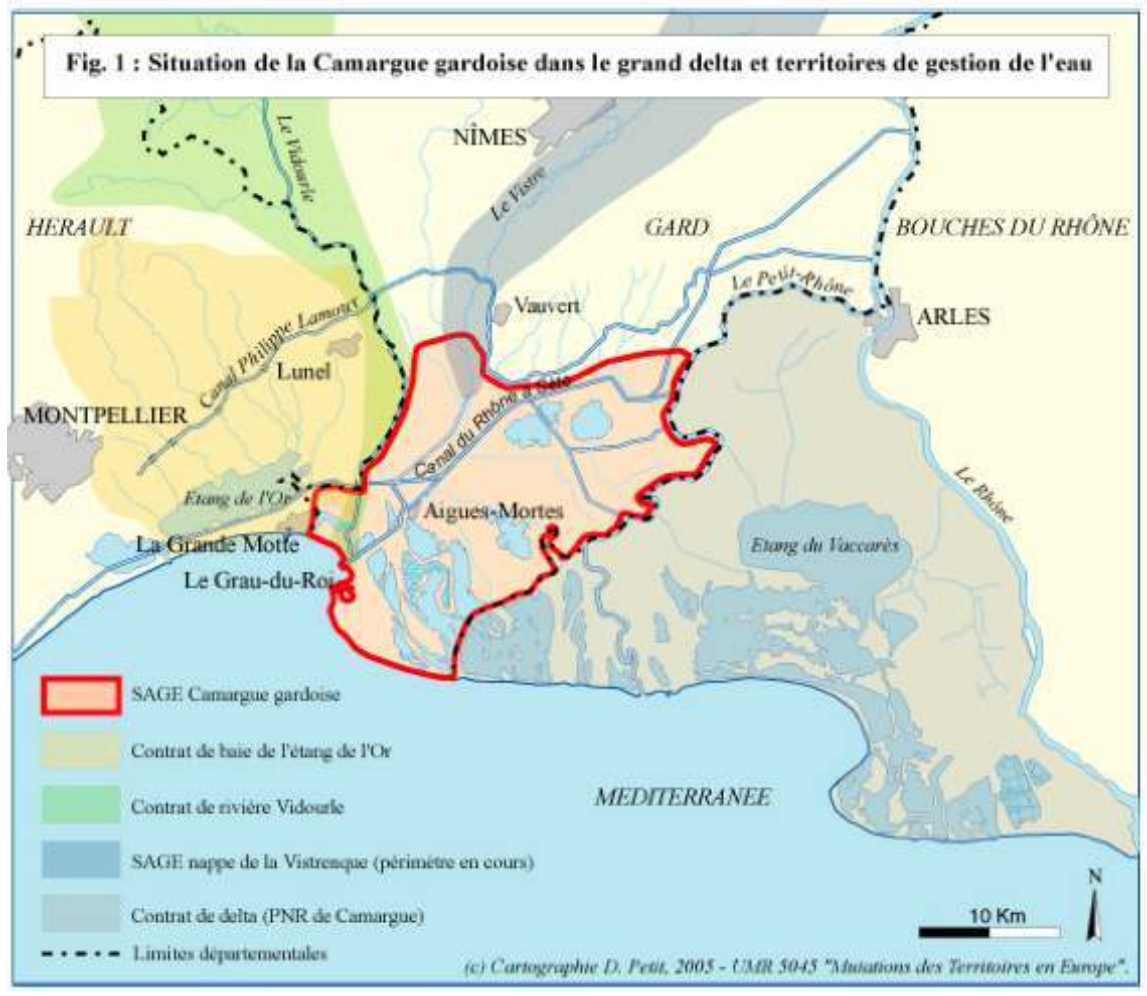

[afficher la carte]

6 Ce patrimoine exceptionnel est original en ce qu'il ne se maintient et n'est dû qu'à l'action anthropique qui s'exerce par la maîtrise de l'eau (Petit D., Arnassant S., 1998, Mathevet R., 2004). En effet, à la spécificité des milieux naturels et des espèces favorisée par la présence d'eaux douce, saumâtre et salée, s'ajoute un fonds culturel et de savoir- 
faire important, basé sur des activités productives ou de loisirs utilisant l'eau: l'exploitation du roseau, appelé localement «la sagne », la viticulture « des sables » au sud, celle des "galets Villafranchiens des Costières de Nîmes" au nord, qui la différencient de la grande Camargue et de ses activités davantage tournées vers les loisirs comme la course camarguaise et les battues aux foulques. Cette diversité confère à cette zone d'étangs et de marais un caractère bien différencié dans le delta rhodanien entre les départements des Bouches-du-Rhône et du Gard. Les traits communs sont l'exploitation des salines, la riziculture, le pâturage des sansouires ${ }^{7}$, la chasse à l'eau, etc.

7 L'homme s'est efforcé de conquérir ces terres humides dès le Moyen Âge, construisant partout digues, fossés, canaux de drainage, polders agricoles ou marais salants. La défense contre la mer, l'assèchement des terres, la mise en valeur halieutique, agricole ou industrielle en sont les raisons récurrentes. La répartition des activités se calque nécessairement sur celle de la salinité des eaux et des sols, commandée par la présence plus ou moins proche de la surface des aquifères salés. Globalement, le degré de salinité présente un gradient nord-sud. L'eau est ainsi soumise à un nécessaire partage entre compétition et coopération, et donne lieu à des pratiques originales, parfois conflictuelles. Une succession de phénomènes originaux, adaptatifs illustrant bien cette combinaison évolutive entre l'environnement naturel et les sociétés humaines, peut ainsi être étudiée (Picon B., 1978, Rivière Honegger A., 1992, Suanez S., Sabatier F., 1999).

\section{Des « infernaulx palus » à leur valorisation, la question du partage et de la gestion de l'eau ne cesse de défier les sociétés locales}

8 Alors qu'en Grande Camargue (Bouches-du-Rhône), la création de la réserve nationale en 1927, consolidée par celle du Parc naturel régional en 1970, donnait une indéniable orientation de conservation, les assèchements s'intensifiaient en Petite Camargue (du côté Gard) dans les années 1960 dans un dessein agricole (installation des rapatriés d'Algérie) et sur le littoral pour la création de stations touristiques nouvelles (mission Racine).

9 Ces nouveaux espaces conquis sur les zones humides accentuèrent les antagonismes entre les usages traditionnels et les nouvelles activités. Ils ont été stigmatisés par les grandes mutations sociales, économiques, techniques et foncières des années 1960 aux années 1980 : vente des marais communaux, extension de la riziculture, mécanisation de la récolte du roseau, multiplication des manades (élevage de taureaux et de chevaux camarguais), privatisation de la chasse, demandes en espaces naturels de loisirs, etc. Au nord, au pied des Costières de Nîmes, l'aménagement du canal Philippe Lamour pour l'irrigation agricole a renforcé, dans le même temps, ces grandes mutations. Sur de vastes espaces, l'arboriculture intensive a supplanté la vigne et les prairies naturelles à partir de la fin des années 1970.

10 Dans ce contexte, les acteurs économiques liés à l'eau se sont positionnés sur des logiques individualistes pour cause d'obsolescence du «Traité des Marais» qui réglait collectivement, depuis 1823, la gestion des niveaux d'eau. En l'absence d'accords possibles entre les différents groupes et les Associations syndicales autorisées, chacun a développé des stratégies cherchant à s'affranchir des contraintes naturelles et sociales pour assurer le devenir et la rentabilité de son activité. L'eau, le principal facteur (avec le climat de 
type méditerranéen et le sel) sur lequel les agents peuvent intervenir est devenue l'objet de maints conflits (Rivière Honegger A., 1992). La méconnaissance de la complexité et de la fragilité des milieux humides méditerranéens, la primauté de la compétition sur la coopération, l'absence de vision globale et de concertation, ont conduit chacun à agir isolément sans une véritable conscience des conséquences de ses stratégies sur la durabilité de la ressource.

11 Ces comportements ont conduit à une gestion de l'eau (circulation et niveaux), de l'espace (cloisonnement, isolement des voisins), et des milieux (chasse), de plus en plus antagoniste, et contraire au maintien de leur qualité et de leur pérennité. Ainsi, la mécanisation de la coupe des roseaux en hiver à l'aide de gros engins détériore-t-elle progressivement le tapis rhizomique des roselières. Les eaux de lavage des terres et de colature de rizières, aboutissant dans les étangs, accroissent l'apport en sel et en matière organique dans ces derniers et contribuent à la disparition des herbiers aquatiques. Le maintien de l'eau en été pour les besoins de la chasse ne permet plus la minéralisation de la matière organique produite par les roselières et les herbiers aquatiques. Les vases noires s'accumulent et le milieu aquatique se trouve fréquemment en état d'anoxie ${ }^{8}$. La charge permanente en eau, sur l'ensemble de la zone, induit une pression sur la nappe salée sous-jacente provocant des remontées salées latérales pendant l'été. En certains endroits, la roselière et les prés sont ainsi remplacés par une sansouire. Le nonrenouvellement des eaux de surface devient néfaste au roseau (Chaigne V., 1987). Au cours des vingt dernières années, la roselière, habitat pour de nombreuses espèces protégées et support ou ressource de plusieurs activités économiques locales, n'a cessé de régresser et surtout de se dégrader.

(estion commune des marais et des étangs. Certains groupes d'usagers représentent des corporations influentes à divers titres, soit qu'ils constituent un secteur économique important (riziculture, chasse privée, sagne), soit qu'ils aient une influence politique forte (pêcheurs, chasseurs), soit encore qu'ils représentent une part non négligeable de la population (chasse communale, sagne dans une moindre mesure). Cette structuration sociale corporative ne remplit plus, dans ce contexte, les fonctions de régulation et de coopération entre les acteurs. Elle est instrumentée par la sphère politique à d'autres fins que la construction d'une nouvelle « entente des marais ».

\section{D'une gestion communautaire devenue houleuse à une gestion contractuelle}

13 A partir des années 1980, des associations locales s'inquiètent de la situation et réclament une protection de la Camargue gardoise. Au début des années 1990, le Conseil général du Gard entreprend de mettre en œuvre une politique de développement local basé sur la protection et la valorisation de la zone (création de réserves naturelles), la maîtrise de la gestion de l'eau et l'amorce d'une ouverture au tourisme sous la forme dite éco-tourisme.

La réussite de cette politique est liée à la recherche de solutions quant à la gestion de l'eau et à l'évolution des mentalités. L'élément fédérateur entre les divers acteurs de terrain est le fort attachement au territoire, qui passe par la sauvegarde de la zone humide et des activités liées à l'eau. Il fait référence aux longues luttes historiques de la population pour garder ses étangs et ses marais, et surtout au ressentiment général lié aux 
derniers assèchements opérés sur plus de 1800 ha de marais au cours des années soixante (Vassas R., Vigneron J., 1980). Reconquérir ces marais perdus reste pour certains un espoir. Les transformer en "espaces de l'eau ", c'est-à-dire les restaurer en champs d'expansion de crue, est évoqué aujourd'hui.

C'est dans ce contexte qu'est créé, en 1993, le Syndicat mixte pour la protection et la gestion de la Camargue gardoise. La mise en œuvre d'une Charte de l'environnement (1995) associée à un SAGE (approuvé en 2001) constitue le pivot de l'action. Les périmètres, choisis sans opposition particulière, sont des périmètres technicoadministratifs définis davantage par rapport aux structures et organismes préalablement existant sur des parties de la zone humide. Ainsi, ils ne prennent pas forcément en compte les véritables échelles fonctionnelles de l'hydrologie de la zone et ont laissé quelques zones en dehors du SAGE.

Le Conseil général, acteur clef et moteur principal du processus, mène ses actions soit directement, soit par le biais du syndicat mixte ou de la Commission locale de l'eau (mise en place en 1995) qui jouent le rôle de relais sur le terrain. Les communes s'impliquent peu mais restent présentes. Les acteurs de l'eau se sont peu à peu réappropriés la gestion de l'eau. La Région reste en dehors de cette action essentiellement pour des raisons politiques. Le Conseil régional et le Conseil général du Gard relèvent à cette époque de formations politiques différentes. Les rivalités sont très fortes à l'approche des élections régionales de 1998. Par ailleurs, la Région est alors relativement dépourvue d'une réelle politique sur la protection de l'environnement et les questions sur l'eau restent dans la sphère traditionnellement "captée" par la Compagnie du Bas-Rhône Languedoc. Parallèlement à ces nouveaux modes d'action publique, s'affirment de nouveaux territoires.

Un des principes qui ont guidé l'action du Conseil général a été d'instaurer un lieu de débat où les élus aussi bien que les usagers des marais puissent s'exprimer en dehors de toute polémique idéologique ou politique. Le Syndicat mixte et la Commission locale de l'eau ont ainsi joué, dans une certaine mesure, un rôle de tribune libre où chacun peut apporter ses connaissances, ses savoir-faire, exprimer ses interrogations, ses souhaits, soumettre ses interprétations sur l'état des étangs et des marais et sur la situation de son activité par rapport à la gestion de l'eau. Deux raisons peuvent justifier ce principe. Tout d'abord, le fait que les deux niveaux d'intervention locale représentés par le Syndicat mixte (niveau politique et maîtrise d'ouvrage) et la Commission Locale de l'Eau (niveau usagers) permettent de traiter les problèmes à la bonne échelle par rapport à leur fonctionnalité et à leur résolvabilité. L'autre raison, constitutive de ce principe, résulte du fait que les élus et les acteurs se montrent bien plus sensibles au devenir des usages et des traditions que de l'état de la zone humide en elle-même. La stratégie a été d'orienter les pratiques, au bon niveau, en n'ayant que peu recours aux contraintes et de les soutenir par l'octroi de moyens appropriés, dans l'esprit d'une valorisation environnementale des produits et des pratiques adéquates, pour que cela soit profitable au milieu. 
Figure 2 : Niveaux et sous-niveaux de la politique de protection et de développement mise en œuvre en Camargue

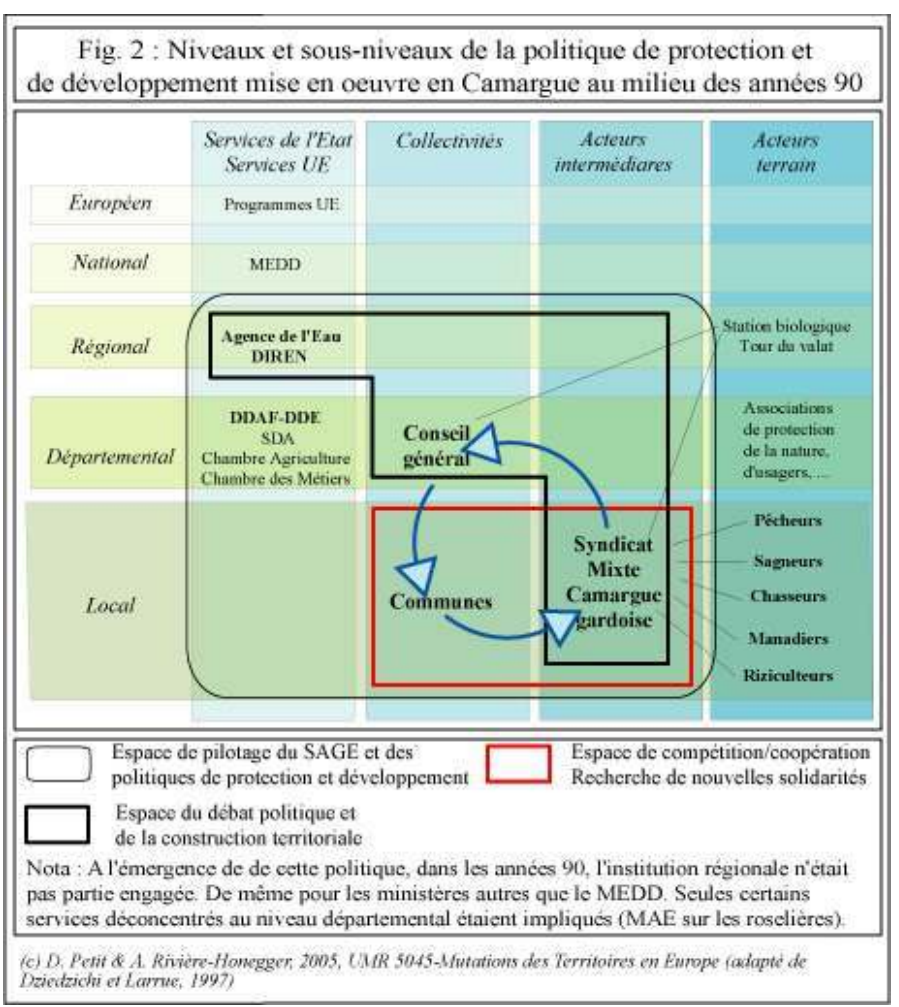

[afficher la carte]

Le temps des débats houleux qui opposaient aménageurs et partisans de la conservation ou d'une exploitation plus raisonnée des zones de marais et d'étangs littoraux est révolu. De nouveaux modes d'aménagement ont été proposés. Des expériences de développement local orientées entre autres vers l'éco-tourisme, la valorisation du patrimoine écologique et historique et la valorisation agri-environnementale se multiplient. Les inondations successives de 2002 et de 2003 ont cependant montré les limites du dialogue. En recentrant les échanges sur la légitime sécurité des biens et des personnes, elles réduisent, à nouveau, la problématique de la zone humide aux questions d'aménagements lourds (rehaussement des digues), mais font surgir aussi la délicate controverse de la pertinence des échelles de décision.

\section{Les crues de 2002 et 2003 : la mise en évidence d'inadéquations territoriales en cas de gestion de crise}

Dans tous les esprits, il y a indéniablement un avant et un après. Aux crues du Vidourle (septembre et décembre 2002) traumatisantes par leur ampleur et leur caractère meurtrier, a succédé les 8 et 9 décembre 2003 une crue du Rhône encore plus imposante ${ }^{10}$. Les chiffres sont évocateurs : 30000 hectares soit les $2 / 3$ du territoire, ont été sous l'eau pendant près d'un mois. Huit communes ont été concernées sur des hauteurs d'eau variant d'une dizaine de centimètres à plus de deux mètres. Cette étendue d'eau correspond à un volume stocké de l'ordre de 300 millions de $\mathrm{m}^{3}$. La question des inondations, sur laquelle avait été largement élaboré le SAGE mais dans un esprit de 
restauration de l'expansion des crues et du décloisonnement des casiers, s'opposant aux endiguements sauvages, est à nouveau au premier plan, occultant tout le reste ${ }^{11}$. L'étude précise de cet événement est riche en enseignements. Il marque une prise de conscience des forces et faiblesses du découpage actuel. Des problèmes de cohérence du périmètre de gestion choisi sont apparus avec acuité. Il semble que le périmètre du SAGE de la Camargue gardoise soit inadéquat pour résoudre cette question qui se situe à une plus vaste échelle, englobant le Rhône (à l'est), le Vidourle (à l'ouest) et des petits cours d'eau de régime méditerranéen, tel le Rhôny et la Cubelle (au nord-ouest). L'outil SAGE, luimême, est-il le bon outil pour cette thématique?

A une cohérence hydrologique ou d'activités liées aux zones humides, il a été préféré, on l'a vu, une logique technico-administrative. La crue, perturbation majeure dans l'historicité du lieu, a rappelé que la Petite Camargue ne peut pas s'en sortir seule. Les seuls exutoires possibles sont le Petit-Rhône, le canal du Rhône à Sète et le Vidourle. Des solutions existent, des blocages aussi. L'Etang de l'Or était le bassin d'expansion originel du Vidourle. Il ne l'est plus pour la partie gardoise. La demande faite aux Salins de remettre en service le Rhône mort a été refusée. En l'absence d'évacuation naturelle possible, les autorités ont alors eu recours à des pompes. Le Syndicat mixte de protection et de gestion de la Camargue gardoise, porteur du SAGE, n'étant pas équipé, il a été fait appel au matériel de pompage agricole des Associations syndicales autorisées (180 millions de $\mathrm{m}^{3}$ ont ainsi été évacués par pompage et par mode gravitaire par le réseau des ASA) puis des pompes hollandaises ont été utilisées en renfort. Tout cela a demandé du temps, a montré l'absence d'un protocole de crise efficient. La question essentielle ${ }^{12}$ : «quels casiers inonder de façon préférentielle ou séquentielle? ? est restée sans réponse. La coordination des acteurs, dans un contexte qui a fortement évolué depuis la décentralisation, tant au niveau des compétences formelles que de celui des moyens et du leadership des dispositifs mis en place, semble bien avoir été, comme partout en Languedoc du reste, le problème majeur. P. Valarié note ainsi que «la multiplication des politiques publiques contractuelles dans les différents domaines, atteste de cette nécessité de dépasser le seul schéma des seules compétences légales et/ou réglementaires face aux problèmes modernes que pose l'action publique» (Valarié P., 2004, p. 60). Il encourage ainsi à adopter une nouvelle conception des échelles d'action qui ne se limitent pas aux découpages hérités ou contraints et qui fasse correspondre les territoires de problèmes avec les territoires de réponse. C'est la question de «l'opérationnalité » des territoires qui est ici posée. On est bien loin de cette situation. Cependant, des clarifications tentent d'être faites.

La fonction de zone d'épandage ${ }^{13}$ n'est ainsi pas remise en cause en Camargue gardoise mais, en contrepartie, des moyens réels ont été demandés pour évacuer l'eau. Les ASA réclament, par exemple, des indemnisations ${ }^{14}$. La CLE a par ailleurs décidé, en décembre 2003, d'établir un règlement d'eau identifiant le rôle de transfert de crue du canal du Rhône à Sète et les modalités de gestion de ses ouvrages en périodes de crises.

La crue passée, des repositionnements sectoriels s'observent. Ils reposent la question de la solidarité spatiale autour de l'eau sur le territoire de la Camargue gardoise et avec les territoires voisins, notamment l'ensemble du Delta camarguais et l'Etang de l'Or, tous deux gérés par d'autres Syndicats mixtes aux objectifs forts semblables ${ }^{15}$. Les événements de 2002 et 2003 montrent bien que les politiques de l'eau ne peuvent faire l'impasse d'une réflexion sur l'articulation entre les différents espaces de décision ${ }^{16}$. A l'espace hydrologique fonctionnel se surimposent d'autres espaces. Ces situations difficiles ont 
mis en évidence des incompatibilités de principe entre le territoire hydraulique défini par le bassin versant et le(s) territoire(s) administratif(s) ou politico-décisionnel(s). L'eau propose une logique qui contredit la gestion des risques telle qu'elle s'applique actuellement. La compréhension des dynamiques territoriales relatives aux risques hydrologiques suppose donc au préalable une définition des risques hydrologiques en jeu (Laganier R., 2003) ; et c'est bien cet effort de définition qui va devoir être fait à présent en Camargue gardoise en lien avec les territoires voisins participant de la genèse des risques. La création, en 2002, de l'observatoire des zones humides et des habitats en Camargue gardoise devrait y contribuer.

\section{Vers la création de nouveaux territoires de l'eau?}

L'eau est bien l'élément fondamental de la structuration de l'espace et de la société en Camargue gardoise. Les utilisations de l'eau constituent le contre-champ du cycle naturel de l'eau. A ce cycle global, s'intègre toute une série de cycles d'utilisation (cycles agricole, des loisirs, urbain, de la navigation...) parfois complémentaires, mais le plus souvent pas ou peu compatibles entre eux et avec le cycle naturel de l'eau. Tous ces cycles doivent faire l'objet d'une double évaluation: celle de la rentabilité économique et celle de l'insertion entre risques liés à l'eau (inondation, sécheresse) et impacts liés aux aménagements. Il n'y a pas de discontinuité entre la ressource naturelle et ses champs d'application: le tout forme un même ensemble qui doit être géré comme tel. On ne peut manquer ainsi de s'interroger sur le bien fondé du périmètre et des échelles de décision qui font, aujourd'hui encore, fi de la notion de système deltaïque - afin d'élaborer un programme d'intervention à l'échelle de territoires opérationnels !

La mise en perspective, à travers l'espace et le temps, des différentes modalités de rapports entre l'environnement naturel et le développement des sociétés humaines, permet de repérer des périodes de permanence relative et des moments de rupture. Ces ruptures provoquent des mutations qui mettent en évidence les préoccupations successives par rapport à l'environnement, l'évolution des représentations sociales, et expliquent les différentes pratiques de la nature. L'exemple de la Camargue gardoise illustre bien cette combinaison évolutive: des relations interactives et rétroactives, multiples et complexes, internes et externes structurent et dynamisent ce système; un système géographique, plus ou moins ouvert, plus ou moins performant selon les périodes de son histoire, plus ou moins résistant ou adaptatif devant les mutations, où des phases néguentropiques et entropiques se succèdent en un même lieu. L'origine des phases d'entropie est historiquement plutôt liée à des facteurs extérieurs au système. C'est le cas, en particulier, lors des grandes mutations économiques (creusement du canal, mécanisation de l'agriculture...). Par contre, il semble que la phase entropique contemporaine ait une double raison. On relève des causes internes qui se rattachent à l'incapacité des groupes sociaux à s'organiser et à envisager une solution, en vue d'un nouveau mode de gestion et d'organisation du territoire. Un mode à trouver qui devra garantir la renouvelabilité de la ressource et le développement, face à l'invention de «nouveaux usages concrets et de nouvelles fonctions imaginaires » (Viard J., 1990, p. 25). On observe également des causes externes aux menaces qui pèsent sur ce territoire (tourisme, spéculations immobilières...). Elles s'inscrivent dans des logiques de développement nationales (schéma d'aménagement du littoral languedocien). Elles sont sans doute les plus prégnantes. Le paradigme espace-temps se présente bien comme une 
clé de lecture et d'analyse à côté de celui de nature-société. Ils peuvent être utilement complétés par celui qui rend compte de l'origine des causes des changements d'état, soit la clé endogène-exogène.

Historiquement, on peut montrer, en Camargue gardoise, que chaque période correspond à une configuration particulière de rapports locaux, de systèmes sociaux, de modèles économique ou technologique, d'enjeux environnementaux et, à partir de celle-ci, détermine une construction de l'espace. L'espace et les temporalités, ainsi construits et gérés dans le contexte des configurations que l'on produit, évoluent vers un seuil ou des changements plus ou moins progressifs qui les conduisent vers une nouvelle configuration à moins qu'une perturbation historique n'intervienne. Ce fut le cas dans les années 1960. La mécanisation rapide de la viticulture, rupture d'origine technique exogène et brutale puisqu'en Camargue gardoise, la mécanisation agricole s'est opérée en moins de dix ans, eut pour effet une mutation radicale de l'exploitation des roselières et, conséquemment, du régime hydraulique appliqué aux marais. Jusqu'aux années 1960, la coupe des roselières se pratiquait en été pour fournir un fourrage vert aux chevaux des vendanges. La gestion de l'eau, liée à ce modèle d'exploitation, maintenait une grande partie des marais asséchés afin de pouvoir y pénétrer avec chevaux et charrettes. Le cycle d'utilisation était, dans ce modèle, relativement synchrone avec le cycle naturel du climat méditerranéen. Avec la perte de ce marché, suite à la mécanisation agricole, les récoltants de roseaux se sont tournés vers un autre marché, celui de la «chaume » pour les toitures. Cette mutation/adaptation a trouvé ses ressorts au sein même des exploitants locaux de roseaux. Devant la fin du marché du roseau en vert, ils ont su exploiter les conséquences des assèchements des zones humides du nord de l'Europe et récupérer le marché de la fourniture en roseaux secs pour les toitures des habitations traditionnelles de ces pays. Cette dynamique adaptative peut être qualifiée d'endogène dans la mesure où aucune politique nationale, à l'époque, ne s'était penchée sur le sort de ces quelques « cueilleurs des marais ", perçus en ces temps mythiques du progrès technique comme d'un «autre temps ». Aujourd'hui, donc, les roseaux sont récoltés après la chute des feuilles c'est-àdire en hiver. Les roselières sont maintenues en eau (si possible circulante) en été afin d'en favoriser la croissance. Le nouveau cycle d'utilisation, conforme à ce nouveau modèle d'exploitation, est largement asynchrone vis-à-vis du cycle naturel. Faut-il interpréter ainsi les crues successives comme une perturbation historique (dans ce cas davantage exogène) qui viendrait modifier les configurations en cours? L'analyse de ce passage entre deux " états " ${ }^{17}$, si cela s'avère être le cas, constitue alors une nécessité.

Enfin, en termes de gestion et de recherche de solutions, comme bien d'autres zones humides ou complexes hydrographiques, les marais de la Camargue gardoise illustrent l'un des changements marquants de la fin du XXème siècle. C'est le retour du collectif comme « agent » de nouvelles formes de gestion de l'eau. Ce retour qui s'inscrit dans une évolution des mentalités et des incitations administratives à une échelle beaucoup plus globale (Aspe, 1998), pose la question de la légitimité de négociation et d'action parmi une diversité croissante d'usages et d'activités autour de l'eau. Comment dépasser la prégnance des jeux locaux et des configurations particulières d'acteurs territoriaux? Quelles sont les possibilités de valorisation de ces espaces par une gestion de l'eau appropriée? Une logique horizontale plurifonctionnelle tente de se substituer à une organisation verticale par filière de la gestion de l'eau. Cette aspiration à plus d'«horizontalité » dans la recherche de solutions, soulève la question des solidarités spatiales. L'eau peut-elle être le vecteur d'une construction territoriale à travers une 
intercommunalité de projet qui lui serait liée? L'articulation avec les projets d'aménagement du territoire est balbutiante et, face à des perturbations socialement mal maitrisées, les tentations sectorielles et monofonctionnelles sont encore puissantes.

\section{Conclusion}

Dans la perspective d'un prolongement des recherches et réflexions sur ce thème, un certain nombre de questions se posent, parmi lesquelles celles des échelles d'action et des référents spatiaux, que l'on peut regrouper sous le terme de stratégie territoriale. On définit celle-ci comme le résultat du rapport entre le collectif et le territoire à adopter (Gumuchian et al., 2003). En l'état de notre réflexion et des données rassemblées, quelques postulats peuvent être énoncés.

Il semble tout d'abord utile de rappeler que la définition géographique du territoire sera toujours à géométrie variable selon les thèmes et les différentes échelles abordés.

Ensuite, il est indispensable de considérer l'eau "comme un élément de coexistence coopérative » selon l'expression de Michel Drain (1998, p. 253), et pas seulement comme un élément qui renvoie à des situations analysées en terme de conflits ou de tensions. La Camargue gardoise est un espace géographique en recomposition depuis une dizaine d'années. La recherche de nouvelles modalités de gestion de l'eau, comprise à la fois en tant que ressource et risque, nous apparaît à la genèse de cette recomposition. La dégradation des milieux naturels, le choc des récentes inondations et les différents entre les acteurs sont certes des éléments majeurs de cette recherche; mais il convient de refuser l'enfermement cartésien dans une vision binaire qui se fonderait soit sur la compétition, soit sur la coopération, sans envisager d'autres options. Rejeter le piège de cette alternative signifie reconnaître la complexité socio-spatiale et rechercher de nouvelles formes de solidarités. C'est faire preuve d'inventivité en rendant inséparables et complémentaires les deux facettes du couple compétition/coopération. En effet, de nos observations sur le terrain, il ressort que le jeu subtil entre les forces, tendant les unes à la coopération et pour les autres, établissant la compétition, crée des sous-ensembles territoriaux de solidarité, plus ou moins contraints et plus ou moins construits, mais dynamisant le territoire. La solidarité spatiale peut être comprise alors, non pas comme un état homogène de stabilité incertain, fragilisé à la moindre perturbation, mais comme une dynamique historique d'un territoire.

31 Il s'agit donc aussi d'identifier et d'analyser les activités et expériences porteuses d'innovation en matière de gestion de l'eau et des territoires. Intégrer cette remarque est indispensable à la compréhension des situations mêlant social et environnemental, en particulier dans notre approche qui fait une large part aux approches diachronique et systémique, pour saisir complémentarité et hétérogénéité spatiales et temporelles (Bravard P., 1998, p. 9). L'analyse diachronique de la gestion hydraulique nous permet en particulier d'analyser les jeux d'acteurs en termes de production de gestion et de régulation de l'eau. Elle met en évidence des logiques spatiales et des facteurs de «territorialisation ».

Enfin, n'oublions pas que la norme, en matière de fonctionnement naturel des zones humides, c'est le changement, au rythme des divers cycles annuels (saisons), pluriannuels et des perturbations, exogènes ou endogènes, anthropiques ou « naturelles » bien souvent imprévisibles ! 
lis l'enjeu finalement nous semble ailleurs. Arrivera-t-on à dépasser cette question du territoire pertinent, finalement «mouvante», pour interroger notre appréhension du temps (long terme, intégration des risques, irréversibilité...), notre mémoire collective et la connaissance du développement de la société?

\section{BIBLIOGRAPHIE}

Agence de l'eau RMC, (2003), « Eau et aménagement du territoire en RMC », Guide technique, n8.

Allain S., Emerit A., 2003, « Projets d'aménagement hydraulique, concertation et planification participative de bassin : une approche en terme d'Action Publique négociée ", Natures Sciences Sociétés, Vol. 11, n 3, pp. 255-265.

Arrus R., 2001, « Mutations et réversibilité dans les modes d'usage de l'eau. Bassin occidental de la Méditerranée », Montagnes méditerranéennes, nº 14, pp. 9-18.

Aspe C., 1998, « Le retour de l'Etat et l'argumentaire scientifique dans les conflits pour l'eau », Territoires en mutation, $\mathrm{n}^{\circ}$ 3, Montpellier, Université Paul-Valéry.

Barrue-Pastor M., 2000, « Les temps de la construction sociale de l'environnement : deux siècles de gestion », in Colloque PIREVS 97, Les temps de l'environnement, Editions du CNRS, pp. 343-356.

Bibeault J-F., 2003, « La gestion intégrée de l'eau : dynamique d'acteurs, de territoires et de techniques ", Cahiers de Géographie du Québec, vol. 47, n 132, pp. 389-411.

Bravard J-P., 1998, « Le temps et l'espace dans les systèmes fluviaux, deux dimensions spécifiques de l'approche géomorphologique ", Les Annales de géographie, n 599, Armand Colin, janv./fév., pp. 3-15.

Chaigne V., 1987, Le Roseau (la Sagne) en Languedoc-Roussillon : analyse socio-économique de son exploitation, mémoire de DESS Aménagement rural et développement local, laboratoire de géographie rurale/Université Paul-Valéry, Montpellier III, p. 13.

Clays-Mekdade C., 2001, Les conflits d'aménagement et de gestion du delta du Rhône, perspective sociologique, «Environnement, politiques publiques et dynamique des activités littorales, travaux et questions des sciences sociales ", séminaire PNEC, Mèze.

Drain M. (Dir.), 1998, « Régulation de l'eau en milieu méditerranéen. Risques et tensions, Territoires en mutation, $\mathrm{n}^{\circ}$ 3, p. 253.

Dziedzicki J-M., Larrue C., 1997, Le principe de subsidiarité et la politique de protection des zones humides : Le cas d'une zone humide en France, la Petite Camargue Gardoise, Etude réalisée pour le compte de la DGXII, Direction Recherche, UE Bruxelles, Observatoire de l'économie et des institutions locales, IUP-Université Paris XII (Créteil), 132 p.

Getches D., 1999, « La gouvernance de bassin, Des limites naturelles pour des décisions relatives aux ressources naturelles ", Espaces et société, n 97-98, pp. 111-132.

Gumuchian H. et al., 2003, Les acteurs, ces oubliés du territoires, Paris, Ed. Anthropos.

Herodote, 2003, « Les pouvoirs locaux, l'eau, les territoires », Paris, La découverte, $n^{\circ} 110,188$ p. 
Laganier R., Scarwell H-J., 2003, « Risques hydrologiques et territoires », In : V. Morionaux (Dir.), Les risques, pp. 106-125.

Mathevet R., 2004, Camargue incertaine, Sciences, usages et natures, Ed. Buchet-Chastel, Ecologie, $208 \mathrm{p}$.

Narcy J-B., 2004, «La politique de l'eau face à la gestion des espaces : les Agences de l'eau aux limites de la modernité ", Espace et sociétés, $n^{\circ} 115, n^{\circ} 4 / 2003$, L'Harmattan, « Ambiances et espaces sonores », pp. 179-196.

Petit D., Arnassant S., 1998, Site de la Camargue gardoise fluvio-lacustre : Document d'objectifs Natura 2000, Nîmes, Syndicat mixte pour la protection et la gestion de la Camargue gardoise-Préfecture du Gard, 3 vol.

Petit D., 1997, Les domaines départementaux de la basse vallée du Vistre : Plan de gestion initial, Nîmes, Conseil général du Gard, 142 p. + annexes.

Picon B., 1978, L'espace et le temps en Camargue : essai d'écologie sociale, Le Paradou, Actes Sud, 264 p.

Pradeau Ch., 1999, « Réseaux hydrographiques et organisation de l'espace », L'information géographique, Paris SEDES, $\mathrm{n}^{\circ}$ 1, vol. 63, pp. 3-9.

Rivière-Honegger A. (Dir.), (1992), « Eau et environnement en Petite-Camargue gardoise, bilan et propositions pour la protection et la gestion du patrimoine naturel », Espace rural, $\mathrm{n}^{\circ} 28$, Montpellier, $181 \mathrm{p}$.

Suanez S., Sabatier F., 1999, «Eléments de réflexion pour une gestion plus cohérente d'un système anthropisé : exemple du littoral du delta du Rhône ", Revue de Géographie de Lyon, $\mathrm{n}^{\circ} 1$, pp. 7-25.

Thomas P., 1995, Influence des pratiques rizicoles sur la qualité des eaux du bassin du Scamandre: Perspectives d'avenir, campagne 1995, Nîmes, Syndicat mixte pour la protection et la gestion de la Camargue gardoise-Centre du Scamandre, Mémoire de fin d'études, ESA Angers.

Valarie P., Coeur D., 2004, «Vulnérabilité de la région Languedoc-Roussillon aux crues méditerranéennes : perspectives historiques et enjeux actuels », La Houille blanche, nº, pp. 56-61. Valiron F., 1987, « L'eau et l'aménagement du Territoire » in Loriferne N. (Dir.), 40 ans de Politique de l'eau en France, Paris, Economica, pp. 157-170.

Vassas R., Vigneron J., 1980, « L'aménagement hydro-agricole de la petite Camargue et l'assainissement des marais de la Souteyranne (Gard) », Société languedocienne de géographie, $\mathrm{n}$ 2-3, tome 14, pp. 267-295.

Viard J., 1990, Le tiers espace, essai sur la nature, Paris, Méridiens Klincksieck, 152 p.

Vitali D., 2003, « Le bassin versant comme territoire des possibles », Méditerranée, n 1-2, pp. 29-34.

\section{NOTES}

1. Les ZICO (Zone d'importance pour la conservation des oiseaux sauvages) sont des bases d'inventaire découlant de la Directive européenne 79/409/CEE dite Directive "Oiseaux ». Elles servent de support pour le classement de zones nécessitant des mesures spéciales de protection pour certaines espèces d'oiseaux : les ZPS (Zones de protection spéciale).

2. Notons, à la suite de Geneviève Barnaux, que la convention de Ramsar (1971) est la seule convention au monde portant sur un écosystème spécifique et qui résulte de l'approche 
convergente des ornithologues et des chasseurs. Sa signature marque le retour en grâce des zones humides. Parcs, $\mathrm{n}^{\circ} 49$, mars 2004.

3. ZNIEFF : Zones naturelles d'intérêt écologique faunistique et floristique.

4. MedWet (Mediterranean Wetland) est un programme international qui vise à inverser la tendance à la dégradation des zones humides et à en assurer une utilisation rationnelle.

5. Arrêté du 26 octobre 2004 portant désignation du Site natura 2000 «Camargue gardoise fluviolacustre » (Zone de protection spéciale), lequel comprend une partie de territoire des communes suivantes : Beauvoisin, Le Cailar, Saint-Gilles, Saint-Laurent-d'Aigouze et Vauvert.

6. Ces quatre espèces sont protégées au niveau national et inscrites à l'annexe I de la Directive «Oiseaux ».

7. Sansouire : formation végétale basse, supportant le sel, à base de salicornes principalement.

8. Anoxie, anoxique : caractérise un milieu pauvre ou dépourvu d'oxygène.

9. «SAGE, un outil pour faire face aux défis de l'eau en Camargue gardoise », plaquette + Cdrom Atlas, juin 2004.

10. SAGE, Commission locale de l'eau de la Camargue gardoise, Compte rendu de la réunion du 15 décembre 2003, mairie d'Aigues-Mortes.

11. Notamment la préservation de la nature et les approches «moins interventionnistes possibles ».

12. Entretien avec un gestionnaire du Centre du Scamandre, mai 2004.

13. Les zones humides ont pour propriété écologique de stocker et restituer l'eau permettant ainsi la régularisation des débits des cours d'eau.

14. Syndicat mixte Petite Camargue gardoise, ASA, Les inondations de décembre 2003 en Camargue gardoise, Les ASA, des acteurs majeurs dans la gestion de la crue. Une contribution d'intérêt général. Dossier d'information, 2003.

15. Le SMGEO (Syndicat mixte de gestion de l'étang de l'Or) a lancé officiellement un Contrat de baie le 26 novembre 2004 ! Un contrat de delta a par ailleurs été signé en juin 2003 pour cinq ans en Grande Camargue sur le territoire correspondant à celui du Parc naturel régional de Camargue...

16. Cette problématique fait l'objet de nombreux débats entre scientifiques et gestionnaires en France et ailleurs (Getches D., 1999, Agence de l'eau Rhône Méditerranée Corse, 2003, Allain S., 2003, Vitali D., 2003, Bibeault J.-F., 2003, Narcy J.-B., 2004).

17. Le concept d'état est considéré à la suite de Monique Barrué-Pastor comme participant «à la fois du temporel et du spatial ; à un état correspond un système de fonctionnement social, un système de mise en valeur. C'est à la fois de l'espace et du temps, du naturel et du culturel, qui se traduit par un découpage en différentes entités de mises en valeur qui ont des dynamiques différentes » (Barrué-Pastor M., 2000).

\section{RÉSUMÉS}

Dans la mouvance des grandes mutations des années 1960-1970 en Camargue gardoise et face à l'obsolescence du Traité des Marais qui réglait la gestion collective des niveaux d'eau, les acteurs économiques liés à l'eau se sont positionnés sur des logiques individualistes. L'eau est devenue l'objet de maints conflits avec de graves conséquences sur les ressources et les milieux. La mise en œuvre d'une Charte de l'environnement et d'un SAGE au début des années 1990 a constitué les 
prémices du retour du «collectif» dans la gestion de l'eau. Mais on est encore loin de l'eau comme vecteur d'une construction territoriale à travers une communauté de projets liée à l'eau. Dans ce contexte, les crues de 2002 et 2003 sont apparues comme des révélateurs des inadéquations territoriales en cas de gestion de crise. Elles montrent que les politiques de l'eau ne peuvent faire l'impasse d'une réflexion sur l'articulation entre les différents espaces de décision et de solidarité.

Next to the major changes which occurred in the "Camargue gardoise " during sixties and seventies, and because of the becoming obsolete "Traité des Marais » which was regulating the collective management of water levels, the economic actors dealing with water concerns have been developing individualistic policies. Water has became a source of many conflicts implying serious consequences on resources and natural spaces. The achievement of an environmental chart and of a "SAGE » at the beginning of the nineties were the ground baits for a come back to a collective form of water management. However, it's a long way to consider water as a vector of territorial construction thanks to collective agreements about projects dealing with water. In the following context, the 2002 and 2003 floods have revealed territorial limitations to manage this kind of crisis. They have shown that the water policies have to take inot more seriously the structural links between the different areas of decision and solidarity.

\section{INDEX}

Mots-clés : France, eau, zone humide, Camargue, dégradation de l'environnement, conflit, ressource, développement local, stratégie d'acteurs, Gard

Keywords : water, wet zone, environmental damages, conflicts, resource, local development

\section{AUTEURS}

\section{DANIEL PETIT}

Daniel Petit est chargé de l'élaboration et de la mise en œuvre des programmes environnementaux régionaux à propos des espaces naturels protégés, de la forêt méditerranéenne et des jardins en Languedoc-Roussillon. Sa longue expérience professionnelle au sein de diverses collectivités territoriales et de leur groupement l'a conduit à animer de nombreux séminaires et groupes de travail sur le thème de la gestion des ressources renouvelables - et particulièrement celles des zones humides - avec des partenaires tels que le CIRAD, l'ENGREF, l'INRA ou le CNEARC. Durant les années 1990, il a été la cheville ouvrière du Centre du Scamandre, établissement publique du Syndicat mixte pour la protection et la gestion de la Camargue gardoise, chargé d'une mission de recherche appliquée et de gestion de la zone humide ainsi que de l'éducation à l'environnement. Aujourd'hui, en fin de carrière professionnelle, il entame un travail d'approfondissement dans le cadre d'une thèse. Géographe, doctorant UMR 5045-CNRS et Chargé de mission à l'Agence Méditerranéenne de l'Environnement (AME) du Languedoc-Roussillon. Coordonnées : UMR 5045 « Mutation des Territoires en Europe », Université Paul Valéry, Route de Mende - 34199 Montpellier cedex 5. Tél : 04-67-14-24-43, daniel.petit14@libertysurf.fr

Publications : Petit D., 2003, «La décentralisation chez nos voisins méditerranéens : quelle traduction en matière de protection de la nature ", La Lettre du Réseau des espaces naturels protégés $d u$ L-R, n²9, pp. 5-6 ; Petit D., 2002, « De l'espace protégé au territoire du développement, du réglementaire au contractuel. Expériences d'une réserve naturelle en Pyrénées-Orientales », 
Revue d'Économie Méridionale, $\mathrm{n}^{\circ}$ 200, $4^{\mathrm{e}}$ trim. 2002, Montpellier, CRPEE/UMR5045, Université Montpellier III, pp. 385-406 ; Petit D., 2001, De la protection de la nature au développement local : L'ancrage des réserves naturelles françaises, Mémoire de DEA de géographie, Université Paul-Valéry Montpellier III, 85 p. ; Petit D., 1997, Les domaines départementaux de la basse vallée du Vistre: Plan de gestion initial. Nîmes, Conseil Général du Gard, 142 p. + cartes et annexes. ; Petit D., Arnassant S., 1998, Documents d'Objectifs Natura 2000 : Site de la Camargue gardoise fluvio-lacustre, département du Gard, Nîmes, Préfecture du Gard/Syndicat Mixte pour la Protection et la Gestion de la Camargue gardoise, 1998, 3 vol ; Petit D., Rivière-Honegger A., 2004, « Le difficile retour du "collectif" dans la gestion de l'eau : regards sur 15 années d'efforts en Camargue gardoise », Cybergéo, http:// www.cybergeo.presse.fr/eauville/Petit\&Honegger.htm

\section{ANNE RIVIÈRE-HONEGGER}

Anne Rivière Honegger, en poste jusqu'en 2005 à Montpellier, s'intéresse depuis longtemps aux questions de l'eau et des territoires en milieu méditerranéen. Elle a soutenu en 1990, à Montpellier, une thèse sur l'eau en Camargue. Actuellement, elle dirige un programme de recherche « Société, territoire et gestion des systèmes hydrauliques dans le Midi de la France. Modernité ou anachronisme d'un mode d'organisation collectif ancien : les Associations syndicales autorisées » (DIREN Languedoc-Roussillon, Région Languedoc-Roussillon (appel à projet de recherche). Elle participe au programme CNRS SHS « Eau, Environnement et Société » intitulé «Crises hydrauliques et perceptions du risque environnemental en Méditerranée occidentale » (projet CRIQUE - 2004-2005) coordonné par J.-P. Billaud (Ladyss). Elle contribue aux travaux du groupe « gestion sociale de l'eau » d'Agropolis-Montpellier. Son récent rattachement à Lyon va lui permettre de développer des travaux dans le cadre de la Zone Atelier Bassin du Rhône. Géographe, Chargée de recherche, UMR 5600-CNRS « Environnement, Ville et Société », Université de Lyon III. Coordonnées : UMR 5600 « Environnement, Ville et Société », Université Jean Moulin, 18 rue Chevreul - 69362 Lyon cedex 07. Tél : 047872 44 58, honegger@clubinternet.fr Publications : Ruf Th., Rivière-Honegger A., 2004, coordination et préface d'un numéro de la revue Territoires en mutation, «La gestion sociale de l'eau, concepts, méthodes et applications », $n$ - 12, 260 p. ; Puech D., Rivière-Honegger A., 2002, coordination et préface d'un numéro de la Revue de l'Economie Méridionale, « Eau, territoire et développement », vol. 49, 2-3, n 194-195, janvier, pp. 119-278; Puech D., Rivière-Honegger A., 2001, coordination et préface d'un numéro de la Revue de l'Economie Méridionale, « Multi-usages et gestion de l'eau en Méditerranée », vol 48, n 191, janvier, pp. 179-388 ; Rivière-Honegger A., Ruf Th., 2000, coordination et préface d'un numéro de la revue Territoires en mutation, « Approches sociales de l'irrigation et de la gestion collective de l'eau.

Démarches et expériences en France et dans le monde ", septembre, 230 p. ; Rivière-Honegger A., 1996, coordination et préface d'un fascicule du Bulletin de la Société Languedocienne de Géographie, « La maîtrise de l'eau en Languedoc », fascicule 1-2/1995, mars, 199 p. 